\title{
VARIAÇÃO ESPACIAL E TEMPORAL DOS FATORES LIMNOLÓGICOS EM RIACHOS DA MICROBACIA DO RIO SÃO FRANCISCO VERDADEIRO
}

\author{
LUCIANO C. DE OLIVEIRA ${ }^{1}$, BENEDITO M. GOMES ${ }^{2}$, GILMAR BAUMGARTNER ${ }^{3}$, \\ NYAMIEN Y. SEBASTIEN ${ }^{4}$
}

RESUMO: Com objetivo de caracterizar espacial e temporalmente os riachos da microbacia do rio São Francisco Verdadeiro, Marechal Cândido Rondon - PR, em função de fatores limnológicos, foram estabelecidas seis estações de coleta em três riachos (Curvado, Ajuricaba e Arroio Fundo), sendo uma estação na foz e outra na nascente. As coletas de água foram obtidas entre junho de 2005 e maio de 2006, totalizando 72 amostras. Foram analisadas as seguintes variáveis: temperatura, $\mathrm{pH}$, condutividade elétrica, oxigênio dissolvido, turbidez, material em suspensão, fósforo total dissolvido, ortofosfato, nitrogênio total e nitrogênio amoniacal. De acordo com a análise de variância, houve baixa variabilidade entre as estações de coleta, porém a variação temporal demonstrou a influência do ciclo hidrológico. A análise de componentes principais (ACP) discriminou o sistema com base nos maiores valores registrados para as variáveis limnológicas, ficando separados os períodos influenciados pelas secas e pelas chuvas. A maior parte das variáveis está associada com o período de chuvas, no entanto há concentrações esporádicas de nutrientes na seca e menores valores de oxigênio dissolvido, demonstrando a dificuldade de diluição nesse período, causada pela diminuição da chuva e, consequentemente, do volume de água dos riachos.

PALAVRAS-CHAVE: variáveis físicas e químicas, limnologia, material alóctone.

\section{SPATIAL AND TEMPORAL VARIATION I OF LIMNOLOGICAL FACTORS IN STREAMS OF THE MICRO-BASIN OF THE SÃO FRANCISCO VERDADEIRO RIVER}

\begin{abstract}
In order to characterize spatially and temporarily streams of the São Francisco Verdadeiro river micro-basin in Marechal Cândido Rondon, State of Paraná, Brazil, according to limnological factors, six sampling stations were established in three streams (Curvado, Ajuricaba and Arroio Fundo) - one station at the mouth of the river and other at the spring of the streams. Water samples were collected between June 2005 and May 2006, totalizing 72 samples. The following variables were analyzed: temperature, $\mathrm{pH}$, electric conductivity, dissolved oxygen, turbidity, suspended matter, total dissolved phosphorus, orthophosphate, total nitrogen and amoniacal nitrogen. According to ANOVA (variance analysis) it was observed low variability among the sampling stations however the temporal variation demonstrated the influence of the hydrologic cycle. The Principal Components Analysis (PCA) discriminated the system based on the largest values registered for the limnological variables separating the periods influenced by droughts and rains. Most of the variables are associated with the rain period; however, sporadic concentration of nutrients in the drought and smaller dissolved oxygen values indicates a dilution difficulty in this period, caused by decrease of rain and consequently decrease of water volume in the streams.
\end{abstract}

KEYWORDS: physical and chemical variables, limnology, aloctone material.

\footnotetext{
${ }^{1}$ Eng ${ }^{o}$ de Pesca, Mestrando do Curso de Pós-Graduação em Engenharia Agrícola, UNIOESTE, Cascavel, Rua Universitária, 2069, Cascavel - PR, Fone: (0XX45) 3220.3175, caetanopesca@ hotmail.com

${ }^{2}$ Eng $^{\mathrm{O}}$ Agrícola, Prof. Doutor, Universidade Estadual do Oeste do Paraná, UNIOESTE, Cascavel - PR

${ }^{3}$ Biólogo, Prof. Doutor, Docente do curso de Engenharia de Pesca, UNIOESTE, Toledo - PR.

${ }^{4}$ Eng $^{\circ}$ de Pesca, Prof. Doutor, Docente do curso de Engenharia de Pesca, UNIOESTE, Toledo - PR.

Recebido pelo Conselho Editorial em: 12-4-2007

Aprovado pelo Conselho Editorial em: 20-10-2008
} 


\section{INTRODUÇÃO}

Os sistemas fluviais são considerados abertos e contínuos, portanto recebem todas as características das áreas adjacentes de sua bacia de drenagem. Essa integração entre os rios e seus ambientes circundantes transforma-os numa unidade funcional mais equilibrada (MARGALEF, 1983). Os rios e riachos são alimentados pela água da chuva, que escoa e percola através do solo, carreando substâncias particuladas e solúveis, influenciando em sua composição química (NETO et al., 1993).

O rio São Francisco Verdadeiro, que se estende desde o município de Cascavel até o reservatório de Itaipu, desaguando entre Pato Bragado e Entre Rios do Oeste, drena uma região de grande importância agrícola, agropecuária e agroindustrial do Brasil, caracterizada pelos elevados índices de produtividade (SOMA, 2002). O uso da terra e as características morfométricas em uma bacia hidrográfica também podem ter grande influência na composição química, física e biológica do rio (RIOS \& CALIJURI, 1995), e quando um ecossistema lótico está contaminado ou eutrofizado, ele está respondendo à tensão recebida pelos ecossistemas terrestres, refletindo o "estado de saúde da bacia hidrográfica" (MARGALEF, 1983).

Nas situações em que predomina o escoamento superficial, a mata ripária atua como barreira aos sedimentos (KLAPPROTH \& JOHNSON, 2000), e a capacidade de transporte torna-se menor que a carga de sedimentos, ocorrendo a sua deposição na interface entre a zona ripária e a faixa vegetativa e a área de cultivo ou pastagem (USDA, 1981). Cerca de 70\% das cargas anuais de nitrogênio e de fósforo que chegam aos rios, são provenientes de atividades agrícolas, sendo os nutrientes que atingem os corpos de água pelos fluxos subsuperficiais ou superficiais (dissolvidos ou agregados às partículas de solo) transportados na forma dissolvida por meio dos fluxos subsuperficiais, onde a mata ripária atua por intermédio de dois mecanismos principais para a redução de nitrato, desnitrificação e assimilação pela vegetação (PEGRAM \& BATH, 1995). O fósforo, por sua vez, é mais frequentemente transportado junto com as partículas do solo e matéria orgânica no escoamento superficial após as precipitações, onde as áreas ripárias são importantes para sua remoção, adsorvido a partículas de solo e matéria orgânica (SIMÕES \& CARDOSO, 2003), porém menos eficiente que na remoção de nitrogênio e sedimentos.

Os rios estão sujeitos a inúmeras perturbações e a biota aquática reage a esses estímulos, sejam naturais, sejam antrópicos (BUSS \& NESSIMIAN, 2003). A detecção dos resultados dos impactos sobre os rios depende do uso de bioindicadores, combinados com variáveis físicas e químicas que regem o funcionamento dos ecossistemas (CALLISTO et al., 2004).

Os objetivos deste trabalho foram monitorar e caracterizar as diferenças entre os parâmetros limnológicos, no âmbito espacial e temporal, em uma microbacia do rio São Francisco Verdadeiro.

\section{MATERIAL E MÉTODOS}

\section{Área de estudo}

A área de estudo corresponde à microbacia do Arroio Fundo, formada pelos riachos Arroio Fundo, Ajuricaba e Curvado, com área de drenagem de aproximadamente 19,7 mil hectares. Nessa microbacia, há cerca de 400 propriedades rurais, e dentre os vários usos múltiplos dessas microbacias destacam-se a captação de água para irrigação, área de lazer pública e corredor de dessedentação. Para a realização das coletas, foram definidas seis estações de amostragem situadas nos três riachos (Figura 1): Curvado $(\mathrm{E} 1=$ Nascente; $\mathrm{E} 2$ = Foz); Ajuricaba $(\mathrm{E} 3=$ Nascente; E4 = Foz), e Arroio Fundo $(\mathrm{E} 5=$ Nascente; E6 = Foz), sendo o posicionamento geográfico das estações apresentado na Tabela 1. 


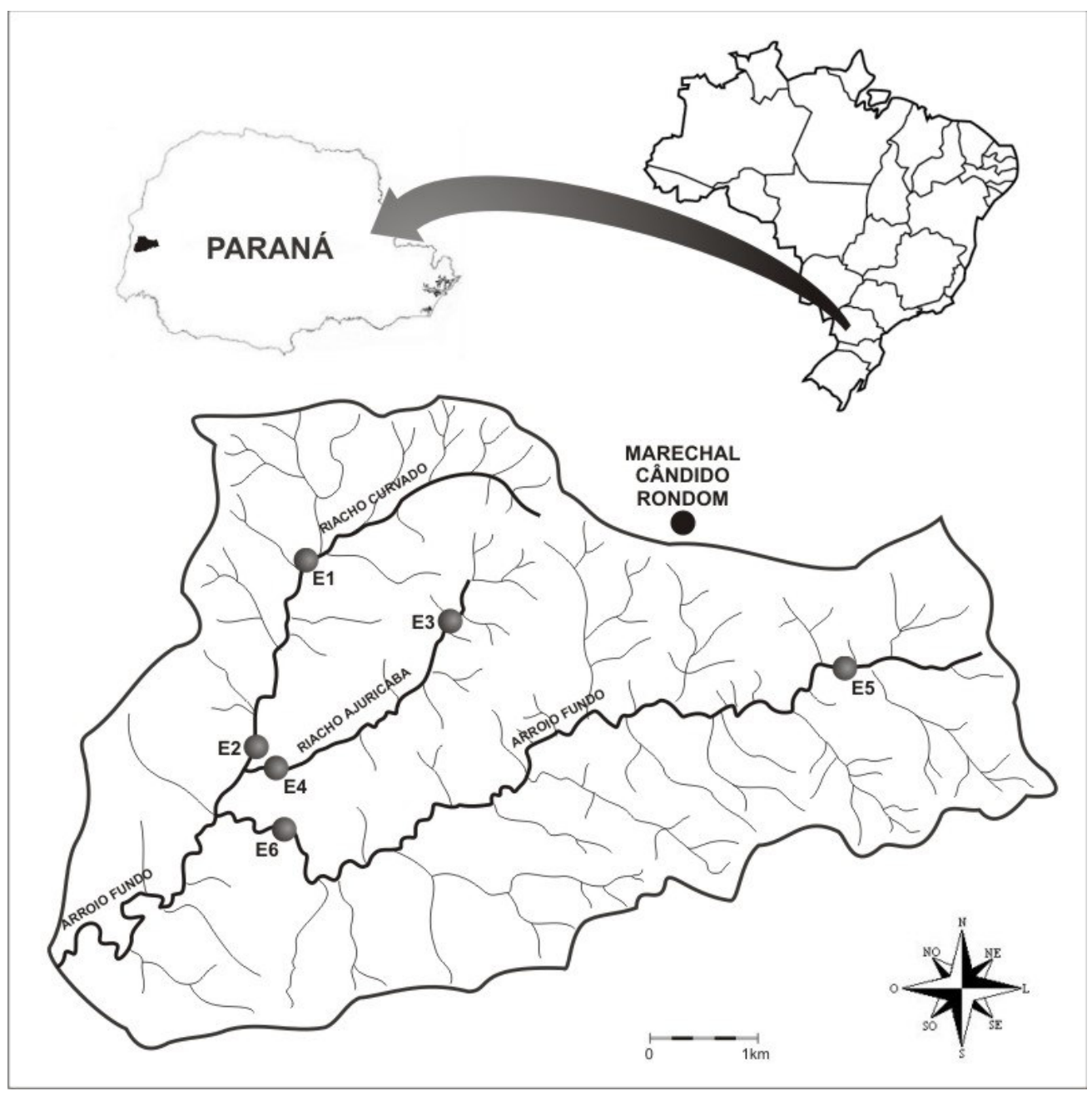

FIGURA 1. Estações de amostragem situadas na microbacia do rio São Francisco Verdadeiro. Sampling stations located in micro-basin of the São Francisco Verdadeiro River.

TABELA 1. Geoposicionamento das estações de coletas. Geoposition station collections.

\begin{tabular}{ccccc}
\hline Riachos & Área & Estação & Município & Posicionamento \\
\hline \multirow{2}{*}{ Curvado } & Nascente & E1 & Marechal C. Rondon & 21J 0788638S/7280166S \\
& Foz & E2 & Marechal C. Rondon & 21J 0787404S/7275769S \\
\hline \multirow{2}{*}{ Ajuricaba } & Nascente & E3 & Marechal C. Rondon & 21J 0791711E/7278627S \\
& Foz & E4 & Marechal C. Rondon & 21J 0787391E/7275728S \\
\hline \multirow{2}{*}{ Arroio Fundo } & Nascente & E5 & Marechal C. Rondon & 21J 0796877S/7277177S \\
& Foz & E6 & Marechal C. Rondon & 21J 0787369S/7274635S \\
\hline
\end{tabular}

\section{Riacho Curvado}

$\mathrm{O}$ ambiente da estação E1 caracterizou-se pelo fundo rochoso, com pequenas áreas de substrato lodoso, com predominância de árvores e arbustos em suas margens. Na estação E2, observou-se fundo pedregoso, e suas margens possuem larga faixa de arbustos, poucas árvores e considerável quantidade de solo desprovido de vegetação.

\section{Riacho Ajuricaba}

Na estação E3, observou-se fundo lodoso, e suas margens apresentam predominância de solo desprovido de vegetação, com pequena faixa de árvores e arbustos. Ambiente inverso foi registrado 
na estação E4, que possui fundo pedregoso, grande faixa de arbustos, poucas árvores e pequena faixa de solo desprovido de vegetação.

\section{Riacho Arroio Fundo}

A estação E5 apresenta fundo pedregoso, suas margens possuem quantidade razoável de árvores e arbustos com faixas de solo desprovido de vegetação em ambas as margens. Situação semelhante ocorre na estação E6, que também apresenta fundo pedregoso, porém em suas margens existe a predominância de árvores e arbustos e pequenas faixas de solo desprovido de vegetação em sua margem esquerda.

A caracterização do solo da microbacia, basicamente como um todo, varia de Latossolo Vermelho e Litossolo Vermelho nas margens dos riachos, com pequenas faixas de Cambissolo háplico.

A precipitação acumulada total nos meses de estudo variou aproximadamente entre $10 \mathrm{~mm}$, nos meses de julho e agosto, a $180 \mathrm{~mm}$, em outubro e novembro, caracterizando estiagem nos meses de inverno e período chuvoso durante a primavera, com variações esporádicas em janeiro, com aproximadamente $100 \mathrm{~mm}$ de precipitação acumulada.

\section{Coleta de água}

As coletas de água foram realizadas mensalmente, no período de junho de 2005 a abril de 2006, quando foram obtidos 5 litros de água em cada estação de amostragem para a análise dos parâmetros limnológicos (físicos, químicos e nutrientes). Após a coleta, as amostras foram acondicionadas em caixas de isopor e transportadas resfriadas, sendo que, no laboratório, as mesmas foram filtradas, obtendo-se as alíquotas filtradas e não-filtradas, que foram acondicionadas em potes plásticos de $500 \mathrm{~mL}$ e preservadas em congelador para análise.

Os parâmetros temperatura da água, oxigênio dissolvido, condutividade elétrica e pH foram medidos no local das amostragens, e os demais parâmetros (material em suspensão, turbidez, nitrogênio amoniacal, nitrogênio total, fósforo total dissolvido e ortofosfato) foram analisados no Laboratório de Limnologia Aplicada da Unioeste, Câmpus de Toledo, seguindo as metodologias propostas por KOROLLEF (1976), MACKRETH et al. (1978) e WETZEL \& LIKENS (2001).

\section{Análises estatísticas}

Para avaliar se ocorreram diferenças significativas entre as variáveis limnológicas nas estações e meses amostrados, foi aplicada a Análise de Variância (ANOVA), e quando essa foi significativa, aplicou-se o teste de Tukey para verificar que estação ou mês de amostragem diferiu dos demais.

Para estabelecer a diferenciação entre os ambientes e os meses amostrados quanto às variáveis limnológicas, foi utilizada Análise de Componentes Principais (PCA) (MANLY, 1985), com os dados previamente transformados em $\log (\mathrm{x}+1)$, para linearizar as relações e reduzir a dimensionalidade. Os eixos retidos para a interpretação foram aqueles que apresentaram autovalores maiores que 1,0, segundo o critério de Kaiser-Gutman-Jackson.

Aos escores dos eixos retidos, foi aplicada a Análise de Variância (ANOVA) para definir diferenças significativas no conjunto de dados. A correlação entre os parâmetros limnológicos foi estabelecida por meio de correlação de Pearson (SOKAL \& ROHLF, 1981) entre as variáveis.

\section{RESULTADOS E DISCUSSÃO}

A condutividade elétrica apresentou ampla variação, entre $7,6 \mu \mathrm{S} \mathrm{cm}^{-1}$, na estação $\mathrm{E} 1$, e $289 \mu \mathrm{S} \mathrm{cm}^{-1}$, na estação $\mathrm{E} 3$, sendo que, espacialmente, essa variável não apresentou diferenças significativas ( $p>0,05)$ (Figura 2A). Porém, mensalmente, diferiu significativamente $(\mathrm{p}<0,05)$, com os meses de novembro $\left(216,9 \mu \mathrm{S} \mathrm{cm}^{-1}\right)$ e dezembro $\left(169,6 \mu \mathrm{S} \mathrm{cm}^{-1}\right)$ apresentando os maiores valores e diferindo de agosto $\left(60,4 \mu \mathrm{S} \mathrm{cm}^{-1}\right)$, que foi responsável pela menor média de condutividade elétrica (Figura 2B). 
Para o $\mathrm{pH}$, entre as estações de amostragem não houve diferença significativa $(\mathrm{p}>0,05)$ (Figura 2C); no entanto, temporalmente, o $\mathrm{pH}$ diferiu significamente $(\mathrm{F}=11,66 ; \mathrm{p}<0,05)$, com os meses de outubro e novembro (ambos 6,79) com menor média, diferindo dos demais meses com maiores médias de $\mathrm{pH}$ (Figura 2D).
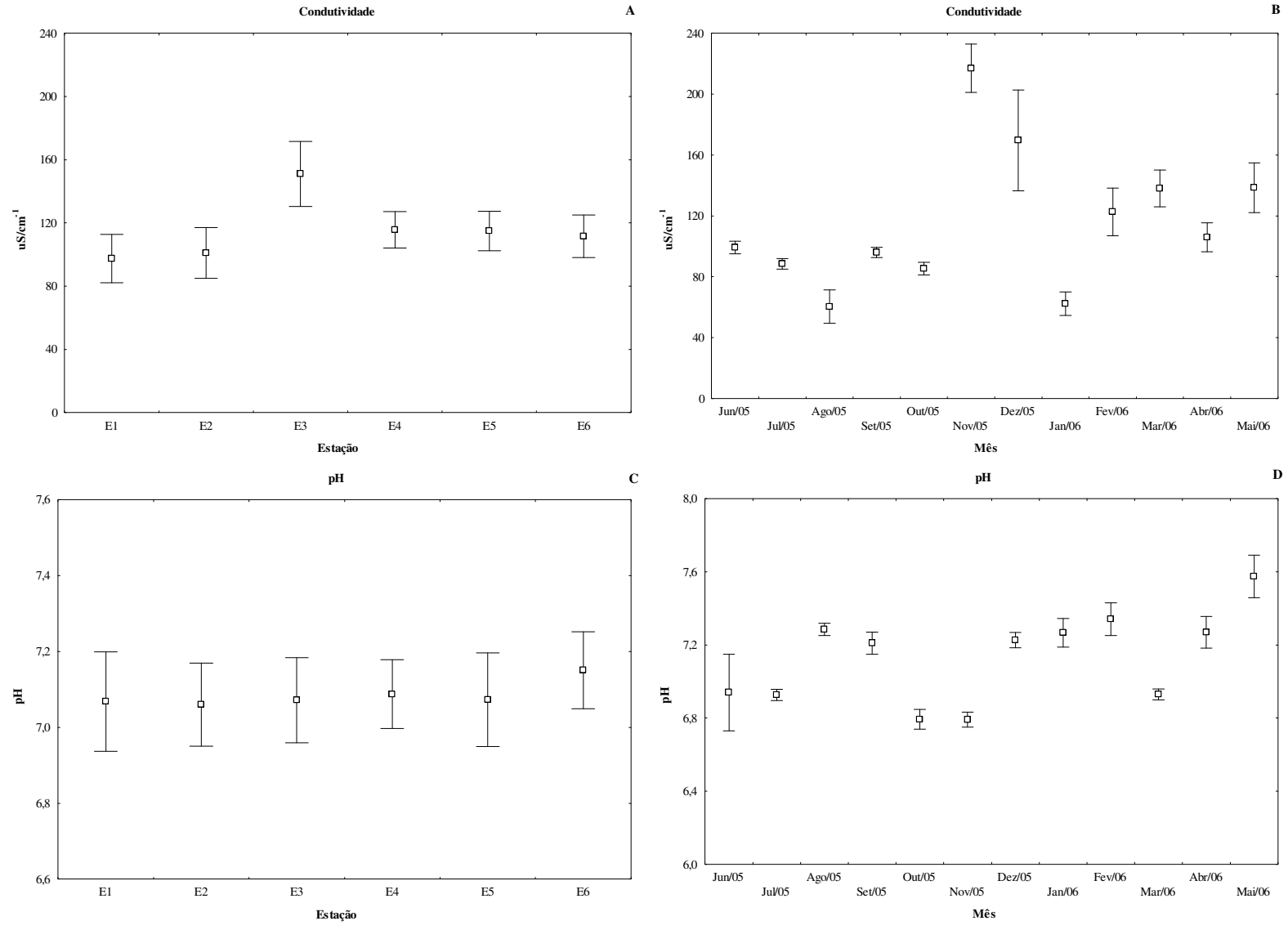

FIGURA 2. (A) Variação espacial; (B) temporal ( $\square$ valores médios; I erro-padrão) de condutividade elétrica, e variações espacial (C), temporal (D) ( $\square$ valores médios; I erro-padrão) de pH obtidos de junho de 2005 a maio de 2006, nas seis estações de amostragem. (A) Change spatial; (B) temporal ( $\square$ average; I standard error) of electric conductivity, and variations spatial (C), temporal (D) ( $\square$ average; I standard error) of pH obtained from June 2005 to May 2006, the six sampling stations.

Cabe destacar que os maiores valores de condutividade elétrica foram registrados em meses chuvosos e nas estações E3 e E4. Resultados semelhantes aos encontrados neste estudo foram registrados por LEITE (2004), em seis rios da bacia do Rio Ji-Paraná, porém esse autor relata que aquela bacia está contida em solos pouco férteis, diferindo da analisada neste estudo. Esse fato diminui a capacidade de fornecer solutos durante o período de menores vazões, quando os rios são alimentados pela água subterrânea e o intemperismo químico é máximo (LESACK, 1993 e MARKERWITZ et al., 2001).

Segundo ESTEVES (1998), em rios tropicais, os valores de condutividade elétrica estão relacionados com as características geoquímicas da região onde se localiza e também com as condições climáticas, o que ficou evidente no presente trabalho, em que os maiores valores desse parâmetro foram associados à maior precipitação e o consequente transporte para o corpo de água.

As pequenas variações espaciais e temporais dos valores de $\mathrm{pH}$ podem ser atribuídas à presença de íons carbonatos e bicarbonatos, que possivelmente estejam formando um sistema tamponante (COLE,1983). A redução dos valores de $\mathrm{pH}$ durante o período de enchente e de cheia 
pode estar relacionado com o aporte de material lixiviado dissolvido. Segundo ESTEVES (1998), esse comportamento dá-se, provavelmente, pela influência da produção de $\mathrm{CO}_{2}$, de $\mathrm{HCO}_{3}{ }^{-}$, e em menor proporção, na forma de $\mathrm{CO}_{2}$ livre, que são responsáveis pelas variações do $\mathrm{pH}$.

A temperatura da água teve amplitude de variação de $12{ }^{\circ} \mathrm{C}$, oscilando entre 16 e $28{ }^{\circ} \mathrm{C}$, e entre as estações de amostragem, a variação da média foi pequena, não diferindo significativamente (Figura 3A). Entre os meses, ocorreu variação sazonal nítida, sendo que essa variável diferiu significativamente $(\mathrm{F}=86,8 ; \mathrm{p}<0,05)$, com os meses de dezembro e janeiro $\left(25,10{ }^{\circ} \mathrm{C}\right)$ (Figura $3 \mathrm{~B}$ ) apresentando as maiores médias, diferindo de maio (menor média $15,2{ }^{\circ} \mathrm{C}$ ). Entre as estações de amostragem, o oxigênio dissolvido apresentou pequena variação, não diferindo significativamente entre as mesmas. Entretanto, os maiores valores foram registrados na estação E5, e a menor média, na estação E1 (Figura 3C). Na Figura 3D, observa-se variação sazonal do oxigênio dissolvido, com os menores valores nos meses mais frios, e os maiores, nos mais quentes. $\mathrm{O}$ mês de novembro (maior valor) diferiu significativamente $(\mathrm{F}=8,33 ; \mathrm{p}<0,05)$ de julho $\left(3,75 \mathrm{mg} \mathrm{L}^{-1}\right)$ e de junho $\left(4,84 \mathrm{mg} \mathrm{L}^{-1}\right)$.

As altas temperaturas verificadas em dezembro e janeiro são características dos rios de regiões tropicais, onde a variação sazonal é pouco acentuada em relação à variação diária (ESTEVES, 1998). Porém, em algumas situações, a sazonalidade anual de temperatura é substituída por estações de alta e baixa pluviosidade.
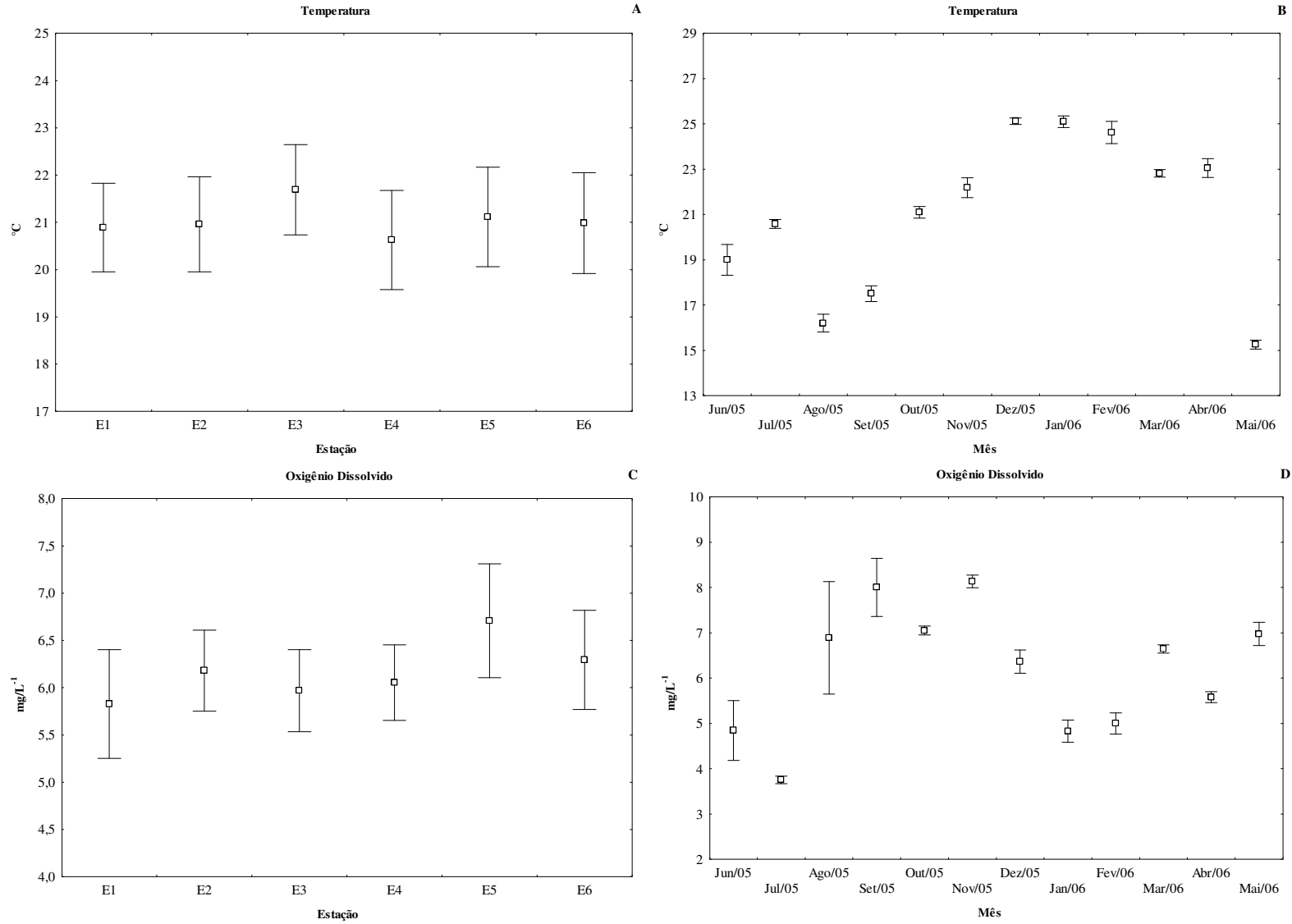

FIGURA 3. (A) Variação espacial; (B) temporal ( $\square$ valores médios; I erro-padrão) da temperatura, e variações espacial (C), temporal (D) ( $\square$ valores médios; I erro-padrão) de oxigênio dissolvido obtidos de junho de 2005 a maio de 2006, nas seis estações de amostragem. (A) Change spatial; (B) temporal ( $\square$ average; I standard error) of temperature, and variations spatial (C), temporal (D) ( $\square$ average; I standard error) of dissolved oxygen obtained from June 2005 to May 2006, the six sampling stations. 
De acordo com a legislação brasileira que define os padrões de qualidade das águas (Resolução CONAMA N ${ }^{\circ}$ 357, de 17 de março 2005), o limite mínimo de oxigênio dissolvido em qualquer amostra é de $5 \mathrm{mg} \mathrm{L}^{-1}$ de $\mathrm{O}_{2}$ para águas doce de classe 2. Foi verificado neste estudo que $23,6 \%$ das amostras estavam abaixo desse limite e, desses, $65 \%$ foram obtidos nos meses de junho e julho, época de estiagem nesses corpos hídricos, o que dificulta a diluição dos poluentes em função da redução do volume de água.

Espacialmente, o material em suspensão não diferiu significativamente $(\mathrm{p}>0,05)$, porém os maiores valores foram registrados nas estações E5 e E6 (15,68 e 12,56 $\mathrm{mg} \mathrm{L}^{-1}$, respectivamente), e o menor valor, na estação E1, com 7,69 $\mathrm{mg} \mathrm{L}^{-1}$ (Figura 4A). Na análise temporal (Figura 4B), verifica-se que o material em suspensão foi maior em outubro (média $42,38 \mathrm{mg} \mathrm{L}^{-1}$ ), diferindo de todos os meses $(\mathrm{p}<0,05)$. Na Figura $4 \mathrm{C}$, observa-se que, entre as estações de amostragem, não ocorreu diferença significativa nos valores de turbidez, porém a estação E5 foi responsável pelos menores valores. Em média, os valores mensais não ultrapassaram $40 \mathrm{UNT}$, exceto no mês de outubro, quando a turbidez alcançou 98,33 UNT (Figura 4D).
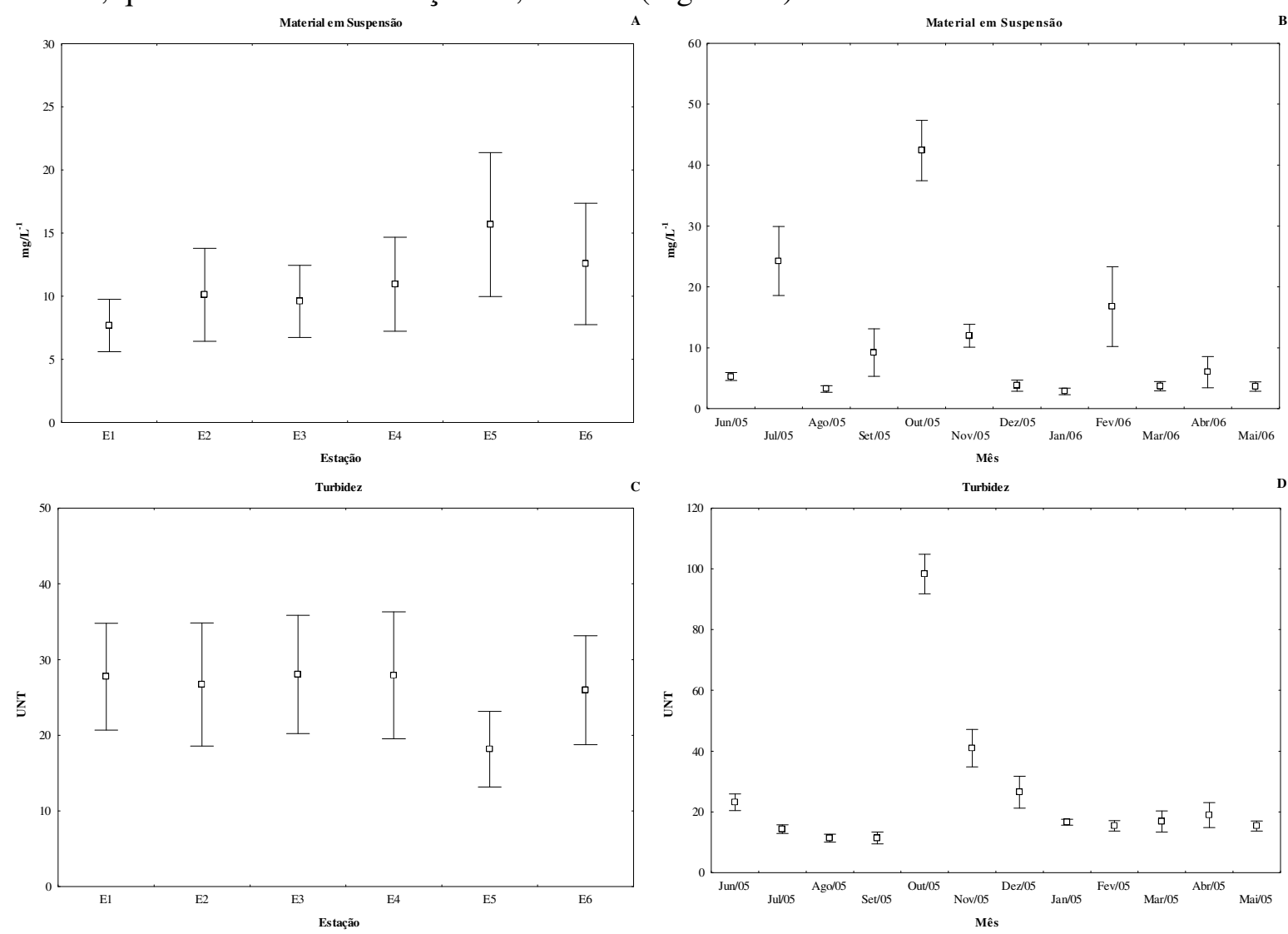

FIGURA 4. (A) Variação espacial; (B) temporal ( $\square$ valores médios; I erro-padrão) do material em suspensão, e variações espacial (C), temporal (D) ( $\square$ valores médios; I erro-padrão) da turbidez obtidos de junho de 2005 a maio de 2006, nas seis estações de amostragem. (A) Change spatial; (B) temporal ( $\square$ average; I standard error) of suspended matter, and variations spatial (C), temporal (D) ( $\square$ average; I standard error) of turbidity obtained from June 2005 to May 2006, the six sampling stations.

A turbidez da água, ao contrário da transparência, reflete de maneira indireta o teor de material orgânico e inorgânico que está em suspensão na água, de forma particulada, portanto é medida inversa da transparência (WETZEL, 2001). Segundo COGO (2005), quando há maior presença de material inorgânico e menor de material orgânico, a origem desse material está ligada à litologia na bacia de drenagem do rio, que apresenta pouca entrada de material orgânico de origem detrital. 
A correlação positiva entre turbidez e material em suspensão $(\mathrm{R}=0,65 ; \mathrm{N}=72)$ demonstra que essas variáveis limnológicas estão associadas aos períodos de chuvas, que proporcionam o carreamento de material sólido para o leito dos riachos, aumentando os valores desses parâmetros. Resultado similar também foi encontrado por FURTADO (2005), no rio Acre, onde a turbidez e o material em suspensão apresentaram correlação positiva $(R=0,90)$ com os maiores valores no período chuvoso.

De maneira geral, em todas as estações de amostragem, as concentrações médias de nitrogênio amoniacal estiveram abaixo de $0,5 \mathrm{mg} \mathrm{L}^{-1}$, exceto na estação $\mathrm{E} 2$, em maio, quando alcançou 5,12 $\mathrm{mg} \mathrm{L}^{-1}$ (Figura 5A). A exemplo das estações, entre os meses, os valores estiveram em média abaixo de $0,5 \mathrm{mg} \mathrm{L}^{-1}$ (Figura 5B), exceto em outubro de 2005 e maio de 2006, quando os valores estiveram acima desse limiar. Entre as estações de amostragem, o nitrogênio total apresentou pouca variabilidade, não diferindo significativamente $(\mathrm{p}<0,05)$ (Figura $5 \mathrm{C}$ ), porém os maiores valores foram observados na estação E3, e as menores, na estação E5. De maneira geral, entre os meses, as concentrações foram aproximadas e abaixo de $0,8 \mathrm{mg} \mathrm{L}^{-1}$ (Figura 5D), exceto em junho e agosto de 2005, que diferiram dos demais meses $(F=6,28 ; p<0,05)$. Como se pode observar na Figura 5, não houve diferenças significativas entre as estações e os meses para esse nutriente. Para rios de classe 2, como os aqui analisados, os valores registrados encontram-se dentro do permitido pela resolução CONAMA No 357 , que possui como limite $2 \mathrm{mg} \mathrm{L}^{-1}$.


FIGURA 5. (A) Variação espacial; (B) temporal ( $\square$ valores médios; I erro-padrão) do nitrogênio amoniacal, e variações espacial (C), temporal (D) ( $\square$ valores médios; I erro-padrão) do nitrogênio total obtidos de junho de 2005 a maio de 2006, nas seis estações de amostragem. (A) Change spatial; (B) temporal ( $\square$ average; I standard error) of amoniacal nitrogen, and variations spatial (C), temporal (D) ( $\square$ average; I standard error) of total nitrogen obtained from June 2005 to May 2006, the six sampling stations. 
Quando comparados os valores encontrados no presente estudo para nitrogênio total com outros ambientes, verifica-se que os valores são menores que os observados em 13 riachos do Estado de São Paulo por LAMPARELLI (2004). Segundo WETZEL (1993) e ESTEVES (1998), os maiores valores de nitrogênio na época de seca podem ser consequência dos processos de decomposição da matéria orgânica, que libera compostos nitrogenados. No entanto, no mês de agosto, dentre as estações localizadas próximo às nascentes, apenas a E3 (riacho Ajuricaba) apresentou redução em direção à estação E4 (foz). As demais apresentaram gradiente longitudinal de aumento nas concentrações em direção às estações de foz.

Segundo FURTADO (2005), os maiores valores, nesse período, estão relacionados com a maior taxa de decomposição em função do baixo nível da água e diminuição do fluxo, que favorece os processos de decomposição da matéria orgânica carreada no riacho.

As concentrações de fósforo total dissolvido entre as estações apresentaram pouca variabilidade, não diferindo significativamente $(p<0,05)$, sendo os maiores valores registrados na estação E2, e os menores, na estação E6 (Figura 6A). Por outro lado, entre os meses, houve diferença significativa $(F=5,04 ; p<0,05)$, com o mês de março diferindo dos demais (Figura 6B). As concentrações de ortofosfato não diferiram espacialmente (Figura 6C) nem temporalmente (Figura 6D), mantendo-se, na maioria das amostras, abaixo de $0,09 \mathrm{mg} \mathrm{L}^{-1}$.
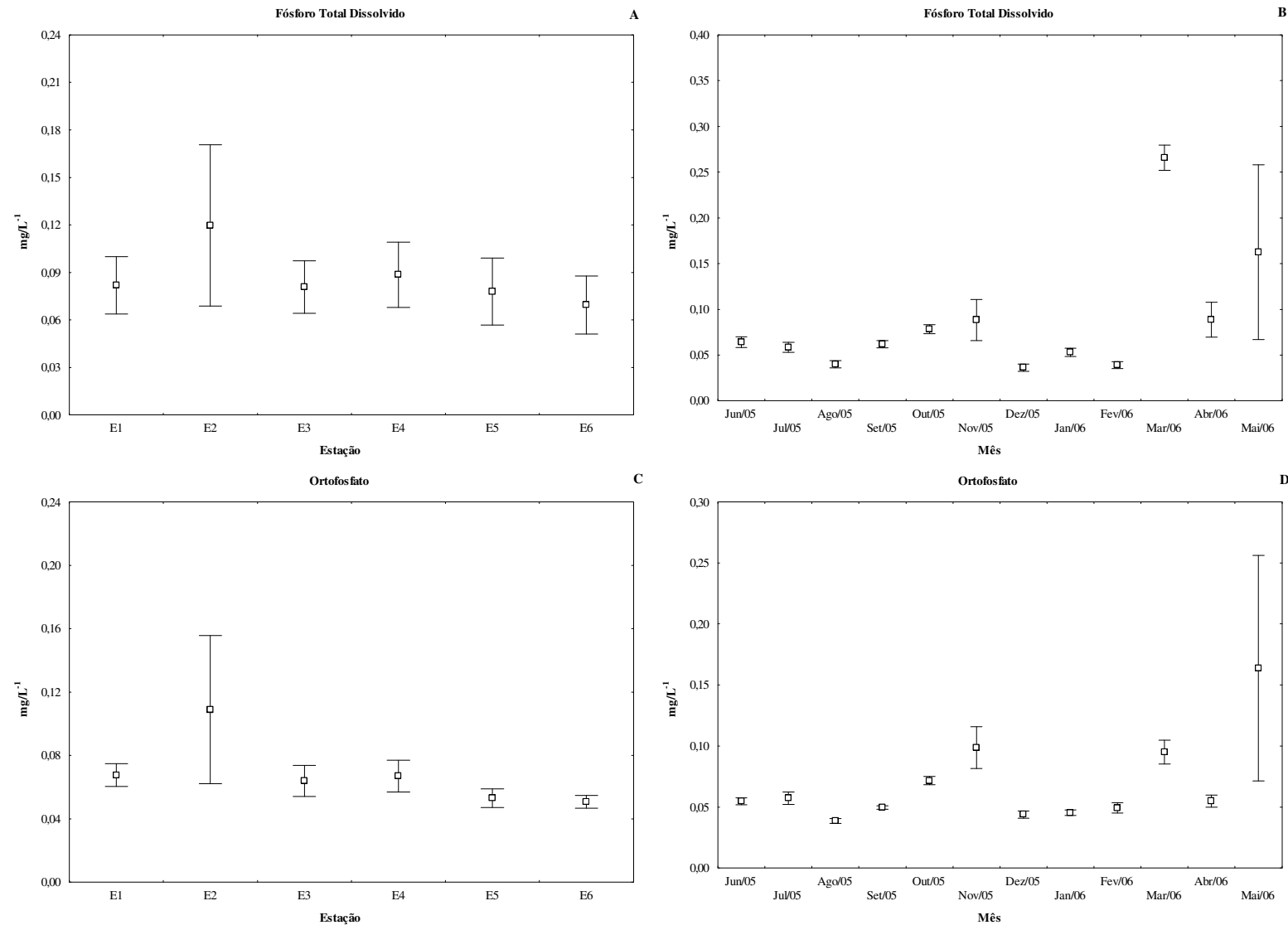

FIGURA 6. (A) Variação espacial; (B) temporal ( $\square$ valores médios; I erro-padrão) de fósforo total dissolvido, e variações espacial (C), temporal (D) ( $\square$ valores médios; I erro-padrão) de ortofosfato obtidos de junho de 2005 a maio de 2006, nas seis estações de amostragem. (A) Change spatial, (B) temporal ( $\square$ average; I standard error) of total phosphorus dissolved, and variations spatial (C), temporal (D) ( $\square$ average; I standard error) of orthophosphate obtained from June 2005 to May 2006, the six sampling stations. 
Os compostos fosfatados presentes nos rios são decorrentes das atividades físiológicas, oriundas da decomposição do ácido fosfórico; essa decomposição ocorre mediante a presença de íons capazes de formar um complexo. Tanto o ortofosfato quanto o fósforo total dissolvido indicam que o corpo do rio apresenta alteração da qualidade por diferentes fontes, sendo uma delas a degradação, e a outra, a atividade fotossintética.

Segundo PAYNE (1986), a concentração do ortofosfato é bastante variável por conta de sua rápida incorporação pelas comunidades aquáticas. No entanto, o fósforo total e o nitrogênio total são os melhores indicadores do conteúdo de nutrientes em qualquer ecossistema (ESTEVES, 1998).

A Análise de Componentes Principais (ACP) revelou que apenas os eixos 1; 2 e 3 apresentaram autovalores maiores que 1,0. Esses três eixos, em conjunto, explicaram 68,7\% da variabilidade dos dados . O primeiro eixo da Análise de Componentes Principais (CP1) apresentou autovalor de 2,7 e explicou $36,7 \%$ da variabilidade, e as variáveis limnológicas que mais contribuíram negativamente para a formação desse eixo foram ortofosfato, fósforo dissolvido e nitrogênio amoniacal. No segundo eixo (CP2, com autovalor de 1,84), contribuíram positivamente a temperatura da água, turbidez e material em suspensão, explicando $18,4 \%$ da variabilidade. No terceiro eixo (CP3, com autovalor de 1,36), as variáveis condutividade elétrica e oxigênio dissolvido contribuíram negativamente para a formação desse eixo (Tabela 2).

TABELA 2. Autovetores das variáveis limnológicas, obtidas por meio da Análise de Componentes Principais, e autovalores dos eixos CP1, CP2 e CP3 (em negrito, estão as variáveis que mais contribuíram para a formação dos eixos). Autovectors limnological of variables, obtained through principal components of analysis of eigenvalues axes CP1, CP2 and CP3 (in bold are the variables that contributed most to the formation of axes).

\begin{tabular}{lrrc}
\hline Variáveis Limnológicas & CP1 & CP2 & CP3 \\
\hline Condutividade & $-0,37$ & 0,35 & $\mathbf{- 0 , 6 5}$ \\
Ph & 0,30 & $-0,46$ & $-0,09$ \\
Temperatura da Água & 0,03 & $\mathbf{0 , 7 5}$ & $-0,01$ \\
Oxigênio Dissolvido & $-0,39$ & $-0,03$ & $\mathbf{- 0 , 6 6}$ \\
Turbidez & $-0,36$ & $\mathbf{0 , 6 0}$ & 0,27 \\
Nitrogênio Amoniacal & $\mathbf{- 0 , 8 1}$ & $-0,18$ & 0,27 \\
Fósforo Dissolvido & $\mathbf{- 0 , 8 1}$ & $-0,25$ & 0,01 \\
Nitrogênio Total & 0,24 & $-0,38$ & 0,33 \\
Ortofosfato & $\mathbf{- 0 , 8 9}$ & $-0,32$ & 0,14 \\
Material em Suspensão & $-0,20$ & $\mathbf{0 , 5 0}$ & 0,46 \\
Autovalores $(\lambda)$ & 2,70 & 1,84 & 1,36 \\
\% de explicação & 36,7 & 18,4 & 13,6 \\
\hline
\end{tabular}

De acordo com a ANOVA aplicada aos escores do eixo 1 (CP1), as variáveis limnológicas diferiram significativamente entre os meses $(\mathrm{F}=17,97 ; \mathrm{p}<0,05)$, sendo que outubro, novembro e março diferem dos demais meses. Ressalta-se que a ordenação desse eixo foi influenciada, principalmente, pelos nutrientes nitrogenados e fosfatados, que também se correlacionaram entre si, nitrogênio amoniacal com fósforo total dissolvido $(\mathrm{R}=0,68 ; \mathrm{N}=72)$, ortofosfato com nitrogênio amoniacal $(\mathrm{R}=0,91 ; \mathrm{N}=72)$ e as formas fosfatadas entre si $(\mathrm{R}=0,83 ; \mathrm{N}=72)$. $\mathrm{O}$ segundo eixo (CP2) também apresentou diferença significativa entre os meses $(F=19,94 ; p<0,05)$, revelando que os meses de agosto e maio diferem dos demais. Os escores do eixo CP3 também diferiram entre os meses $(F=4,93 ; p<0,05)$, porém essa ordenação não foi de maneira nítida, ou seja, os eventos que interferem no meio, estão agindo de forma aleatória. 


\section{CONCLUSÕES}

Baseado nos dados analisados, as variações sazonais de alguns parâmetros limnológicos apresentaram forte correlação com as características naturais, como os períodos chuvosos. As concentrações médias das variáveis limnológicas analisadas estão dentro dos parâmetros propostos pelo CONAMA $\mathrm{N}^{\circ} 357$, porém cabe observar que as maiores concentrações de nitrogênio amoniacal, nitrogênio total, fósforo total dissolvido e ortofosfato não estão relacionadas com nenhum evento natural, tendo em vista que a forte relação entre esses fatores aponta para o aporte de nutriente alóctone por vias difusas ou pontuais.

No que se refere à questão metodológica, este estudo leva-nos a crer que a falta de coleta de dados limnológicos, coincidindo com os picos de cheia, revela a necessidade de ter um plano de trabalho mais flexível, considerando sempre os processos hidrológicos que ocorrem em microbacias, tão como as fontes difusas de poluição.

Apesar de estes dados não permitirem o isolamento dos principais fatores controladores (naturais x antropogênicos) da química dessas águas, as alterações, principalmente de oxigênio dissolvido, devem ser levadas em consideração no contexto da capacidade de autodepuração dos corpos hídricos, já que a vegetação ripária, o manejo do solo e o tratamento de efluentes agroindustriais devem ser maximizados, no intuito de minimizar o aporte de formas nitrogenadas, fosfatadas e sedimentos.

\section{REFERÊNCIAS}

BUSS, D. F.; NESSIMIAN, J. L. Bases conceituais para a aplicação de biomonitoramento em programas de avaliação da qualidade da água de rios. Cadernos de Saúde Pública, Rio de Janeiro, v.19, n.2, p.465-73, 2003.

CALliSTO, M.; GOULART, M.; MEDEIROS, A.O.; MORENO, P.; ROSA, C.A. Diversity assessment of benthic macroinvertebrates, yeasts, and microbiological indicators along a longitudinal gradient in Serra do Cipó, Brazil. Brazilian Journal Biology, São Carlos, v.64, n.4, p.743-55, 2004.

COGO, M.C. O papel dos sedimentos em suspensão no metabolismo de rios de micro e mesoescala no Estado de Rondônia. 2005. 61 f. Dissertação (Mestrado em Ecologia de Agroecossistema) - Escola Superior de Agricultura “Luiz de Queiroz”, Universidade de São Paulo, Piracicaba, 2005.

COLE, G.A. Textbook of limnology. $3^{\text {rd }}$ ed. London: C.V. Mosby Company, 1983. p. 261-81.

ESTEVES, F.A. Fundamentos de limnologia. Rio de Janeiro: Interciência, 1998. 575 p.

CONAMA. CONSELHO NACIONAL DO MEIO AMBIENTE. Resolução n.357, de 2005.

Estabelece a classificação das águas doces, salobras e salinas do Brasil. Diário Oficial da União, Brasília (DF), 2005.

FURTADO, C.M. Caracterização limnológica e avaliação da qualidade da água de um trecho urbano do rio Acre, Rio Branco - AC, Brasil. 2005. 58 f. Dissertação (Mestrado em Ecologia e Manejo de Recursos Naturais) - Universidade Federal do Acre, Rio Branco, 2005.

KLAPPROTH J.C.; JOHNSON, J.E. Understanding the science behind riparian forest buffers: effects on water quality. Virgínia: Virginia Cooperative Extension, Publication Virginia Polytechnic Institute and State University, 2000. p.420-51.

KOROLEFF, F. Determination of nutrients. In: GRASSHOFF, K.; ALMGREEN, T. (Ed.). Methods of seawater analysis. Weinhein: Verlag Chemie, 1976. 317 p.

LAMPARELLI, M. C. Grau de trofia em corpos d'água do Estado de São Paulo: avaliação dos métodos de monitoramento. 2004. 235 f. Tese (Doutorado Ecossistemas Aquáticos e Terrestres) Instituto de Biociências, Universidade de São Paulo, São Paulo, 2004. 
LEITE, N.K. A biogeoquímica do rio Ji-Paraná, Rondônia. 2004. 44 f. Dissertação (Mestrado em Ecologia de Agroessistemas) - Escola Superior de Agricultura "Luiz de Queiroz", Universidade de São Paulo, Piracicaba, 2004.

LESACK, L.F.W. Export of nutrients and major ionic solutes from a rain-forest catchment in the central Amazon basin. Water Resources Research, Washington, v.29, n.3, p.743-58, 1993.

MACKERETH, F.J.H.; HERON, J.; TALLING J.F. 1978. Water analysis: some methods for limnologists. Kendal: Freshwater Biological Association. Titus Wilson \& Son, 1978. 120 p. (Scientific Publication, 36)

MANLY, B.F.J. The statistics of natural selection. In: Spurious test results due to isolation by distance. London: Chapman \& Hall, 1985. p.186-95.

MARGALEF, R. Limnologia. Barcelona: Omega, 1983. 1.010 p.

MARKEWITZ, D.; DAVIDSON, E.A.; FIGUEIREDO, R.D.O.; VICTORIA, R.L.; KRUSCHE, A.V. Control of cation concentration in stream waters by surface soil processes in Amazonian watershed. Nature, London, v.410, n.6830, p.802-5, 2001.

NETO, M.S.S.; ALVES, R.; FIGUEIREDO, A.C.; HINO, K. Caracterização hidrogeoquímica da bacia do Rio Manso, Cuiabá - MT. Acta Limnologica Brasiliensis, Botucatu, v.6, p.230-44, 1993.

PAYNE, A.I. The ecology of tropical rivers and reservoirs. Chichester: John Wiley, 1986. 301 p.

PEGRAM, G.C.; BATH, A.J. Role on non-point source in the development of a water quality management plan for the Mgeni River catchment. Water Science Technology, Sweden, v.32, n.5-6, p.175-82, 1995.

RIOS, L.; CALIJURI, M.C. A bacia hidrográfica do Ribeirão do Feijão: Uma proposta de ordenação das sub-bacias através de variáveis limnológicas. Acta Limnologica Brasiliensis, Botucatu, v.7, p.151-61, 1995.

SIMÕES, L.B.; CARDOSO, L G. Avaliação das larguras de matas ripárias para controle da poluição difusa. Energia na agricultura. In: R. HENRY. Ecótonos nas interfaces dos ecossistemas aquáticos. São Carlos: RIMA, 2003. p.339-49.

SOKAL, R.R.; ROHLF, F.J. Biometry. $2^{\text {nd }}$ ed. New York: W.H. Freeman and Company, 1981. $859 \mathrm{p}$.

SOMA. SOLUÇÕES EM MEIO AMBIENTE . Plano básico ambiental. Curitiba: [s.n.], 2002. $148 \mathrm{p}$.

U.S. DEPARTAMENT OF AGRICULTURE. Riparian forest buffer: function and design for protection and enhancement of water resources. Pennsylvania, 1991. 24 p.

WETZEL R.G. Limnology. Lake an river ecosystems. $3^{\text {rd }}$ ed. San Diego: Academic Press, 2001. $1.006 \mathrm{p}$.

WETZEL, R.G. Limnologia. Lisboa: Fundação Calouste Goulbekian, 1993. 919 p.

WETZEL, R.G.; LIKENS, G.E. Limnological analyses. $2^{\text {nd }}$ ed. New York: Springer-Verlag, 2001. $391 \mathrm{p}$. 